Zeynep Oruç, Senar Ebinç, Ziya Kalkan, Muhammet Ali Kaplan, Mehmet Küçüköner, Zuhat Urakçı, İdris Oruç, Abdurrahman Işıkdoğan

Faculty of Medicine, Department of Medical Oncology, Dicle University, Diyarbakır, Turkey

\title{
COVID-19 infection in cancer patients: the effect of Hepatitis B immunization
}

\section{Corresponding author:}

Zeynep Oruç, Dicle University,Faculty of Medicine,Department of Medical Oncology, Billstreet.SUR, 21280

Diyarbakır, Turkey

e-mail: zeynep44oruc@hotmail.com

Medical Research Journal 2021;

Volume 6, Number 2, 86-93 DOI: 10.5603/MRJ.a2021.0018

Copyright (C) 2021 Via Medica

ISSN 2451-2591

e-ISSN 2451-4101

\begin{abstract}
Introduction: To investigate the clinical characteristics and outcomes of cancer patients with COVID-19 infections and evaluate the effect of hepatitis B immunization status on susceptibility to COVID-19 infection and mortality risk.

Materials and methods: The records of 1,515 patients who presented to the Medical Oncology clinic between March 2020 and December 2020 were analysed retrospectively. The demographic and clinical characteristics and laboratory findings of cancer patients with (case group) and without (control group) COVID-19 infection were compared.

Results: Of the 1,515 patients, 153 (10.1\%) had been diagnosed with COVID-19, and the median age of cancer patients with COVID-19 infection was 53.9 (range; 18-82) years. The most common types of cancer were breast cancer (26.2\%), gastrointestinal system cancers (22.3\%), genitourinary-system cancers (16.5\%) and lung cancer (15.5\%). The presence of metastatic disease [hazard ratio (HR): 0.09, 95\% Cl (0.01-0.83), $(p=0.03)$ ] and receipt of palliative chemotherapy in the cancer patients with COVID-19 infections [HR: 0.1, $95 \% \mathrm{Cl}(0.01-0.69),(p=0.02)]$ were identified as prognostic factors in multivariate analysis as univariate analysis did not indicate palliative treatment as a prognostic factor. When the case group and control groups were compared in terms of hepatitis B immunization status ( $p=0.24)$, no statistically significant difference was identified between the two groups. Furthermore, hepatitis B immunization status $(p=0.37)$ were not found to be associated with COVID-19-related mortality risk.

Conclusion: Hepatitis B immunization status were not associated with the risk of COVID-19 transmission and mortality. The present study identified the presence of metastatic disease and palliative chemotherapy as negative and positive prognostic factors, respectively.

Key words: COVID-19 infection, hepatitis B, mortality, immunization
\end{abstract}

Med Res J 2021; 6 (2): 86-93

\section{Introduction}

Cancer patients have been identified as a highrisk patient group during the COVID-19 pandemic [1-3]. SARS-CoV2 (severe acute respiratory syndrome-coronavirus-2) infection is associated with poorer outcomes in cancer patients than in those without cancer, due to associated advanced age, frailty, comorbidities (hypertension, diabetes, cardiac and pulmonary diseases, etc.), malnutrition and treatment-related immunosuppression [3], and there is a further risk of COVID-19 infection due to the associated therapeutic applications and frequent hospital visits [4].
There is a difference in COVID-19 infection and mortality rates between age groups. COVID-19 infection and mortality rates are very low in the population between the ages of 0 and 20 years, in contrast to the adult population, and symptoms are milder among infected children and adolescents [5]. Several hypotheses have been put forward to explain this finding, the first of which claims that the immune system of adolescents and children is not as mature as in adults, and so the immune response against the virus is not exaggerated. The second is that childhood vaccines, such as those for mumps, rubella, poliomyelitis, hepatitis $A$, hepatitis $B$ and varicella, protect SARS-CoV-2 through cross-reactive antibodies [6, 7]. For example, it has been shown 
that in developing countries where the BCG vaccine is included in the routine vaccination program, there is a lower incidence and better clinical outcomes of COVID-19 than in developed countries without routine $B C G$ vaccination $[8,9]$. That said, the role of childhood vaccines in COVID-19 is uncertain and requires further research.

Do the presence of HBV (hepatitis B virus) infection and HBV antibody seropositivity affect clinical outcomes in cancer patients with COVID-19 infections? The answer to this question is not yet known. To the best of the authors' knowledge, there has been no study to date investigating the relationship between COVID-19 and hepatitis $B$ immunization status among cancer patients nor non-cancer patients.

To address the abovementioned hypothesis, the present study investigates the clinical characteristics and outcomes of cancer patients with COVID-19 infections in the authors' oncology centre and examine the relationship between hepatitis $B$ immunization status and the risk of COVID-19 transmission and mortality.

\section{Materials and methods}

The files of all cancer patients who presented to the Dicle University Faculty of Medicine Medical Oncology between March and December 2020 were analysed retrospectively. Information on the demographic and clinical characteristics, treatments, and laboratory findings of the patients (history of COVID-19; COVID-19 PCR test results; HbsAg, Anti-HBs, and Anti-Hbc IgG levels) were obtained from the hospital's file registry system.

Patients with a laboratory-confirmed SARS-CoV-2 infection upon presentation to the pandemic hospital, i.e., patients with a positive PCR test, were accepted as COVID-19 cases. Those who were clinically diagnosed with COVID-19 but who had a negative PCR test were excluded from the study. Cancer patients diagnosed with COVID-19 (positive PCR test) were defined as the case group, while cancer patients not diagnosed with COVID-19 were defined as the control group. The clinical and laboratory findings and survival outcomes of both groups were evaluated.

Of the 1,515 patients who presented to the oncology clinic, 153 were diagnosed with COVID-19 infection during the pandemic. 1,454 of the 1,515 patients had hepatitis $B$ panel tests. But the clinical data of 103 patients with COVID-19 infection were accessed from the archive system, among which the hepatitis B panel tests of 92 were available. 1362 patients, except 92 patients who were diagnosed with COVID-19 infection were defined as the control group. All statistical analyses were made based on these numbers of patients. The patients were categorized according to age, gender, presence of comorbidities, type of cancer diagnosis, disease stage (metastatic/non-metastatic), therapeutic applications (adjuvant/neoadjuvant therapy, palliative therapy and non-receipt of therapy), therapeutic agents (chemotherapy, immunotherapy, tyrosine kinase inhibitors, anti-hormonal therapies), and hepatitis B immunization status (vaccinated, resolved infection, isolated anti-HBc Ab (+), non-immunized and HBsAg-positive), and the case and control groups were compared based on these parameters. The case groups were further categorized into survivors and non-survivors.

\section{Statistics}

A statistical assessment of the data was made using PASW Statistics (Version 18.0. Chicago: SPSS Inc.). Descriptive statistics were used to evaluate patient characteristics and the frequency of parameters, a Student-t-test was applied to normally distributed numerical variables; Chi-square, Fisher's exact and Mann-Whitney $U$ tests were applied for the analysis of non-normally distributed or non-parametric variables, and a logistic regression analysis was made. A multivariate analysis was carried out using the Cox model. The confidence interval of $95 \%$ and $p$-value of the significance of $<0.05$ were accepted.

\section{Results}

Of the 1,515 patients who presented to the oncology clinic, 1,454 patients had hepatitis B panel tests. The study included a total of 1,454 patients, comprising 763 (52.5\%) men and 691 (47.5\%) women whose data could be accessed. During the pandemic, 153 (10.1\%) patients were diagnosed with COVID-19 infection. Of the 153 patients diagnosed with COVID-19, 18 (11.7\%) died. The general characteristics of $103(6.8 \%)$ patients with fully accessible data and a diagnosis of COVID-19 infection are presented in Table 1. The median age of the cancer patients with COVID-19 infection in the present study was 53.9 (range; 18-82) years. Of the patients, $51 \%$ ( $\mathrm{n}: 52$ ) were women, and there was a history of comorbidities in $42.7 \%$ of the patients. The most common types of cancer among the COVID-19-positive patients were breast cancer (26.2\%), gastrointestinal system cancers (22.3\%), genitourinary system cancers (16.5\%) and lung cancer (15.5\%). There was no statistically significant difference in mortality risk by age, gender, and comorbidities among patients with COVID-19 infection. Regarding the type of primary diagnosis, there was also no statistically significant difference in mortality risk $(p=0.76)$.

Metastatic disease was recorded in $60.2 \%$ (n: 62) of the patients, and the rate of metastatic disease was 
Table 1. Mortality risk in patients diagnosed with Covid 19 according to disease and treatment characteristics

\begin{tabular}{|c|c|c|c|c|c|}
\hline & $\mathrm{N}(\%)$ & Non-survivor (N\%) & Survivor (N\%) & HR $(95 \%$ Cl) & P-value \\
\hline All patients & 103 & 18 (17.5\%) & 85 (82.5\%) & & \\
\hline Age (median, yrs.) & $53.9(18-82)$ & $51(18-80)$ & $54(18-82)$ & $0.98(0.95-1.02)$ & 0.51 \\
\hline Gender & & & & $0.55(0.19-1.56)$ & 0.26 \\
\hline Male & $51(49 \%)$ & $11(61.1 \%)$ & $40(47.1 \%)$ & & \\
\hline Female & $52(51 \%)$ & $7(38.9 \%)$ & 45 (52.9\%) & & \\
\hline Comorbidities & & & & $1.45(0.52-4.03)$ & 0.46 \\
\hline Yes & $44(42.7 \%)$ & $9(50 \%)$ & 35 (41.2\%) & & \\
\hline No & $59(57.3 \%)$ & $9(50 \%)$ & $50(58.8 \%)$ & & \\
\hline Diagnosis & & & & & 0.76 \\
\hline Brain & 4 (3.9\%) & $1(5.6 \%)$ & $3(3.5 \%)$ & $1.66(0.07-37.72)$ & 0.74 \\
\hline GUS & 17 (16.5\%) & $1(5.6 \%)$ & $16(18.8 \%)$ & $0.31(0.16-5.95)$ & 0.43 \\
\hline GiS & $23(22.3 \%)$ & $6(33.3 \%)$ & $17(20 \%)$ & $1.76(0.17-18.32)$ & 0.63 \\
\hline Soft tissue & 7 (6.8\%) & $1(5.6 \%)$ & $6(7.1 \%)$ & $0.83(0.04-16.99)$ & 0.90 \\
\hline Breast & 27 (26.2\%) & $3(16.7 \%)$ & $24(28.2 \%)$ & $0.62(0.05-7.31)$ & 0.70 \\
\hline Lung/pleura & $16(15.5 \%)$ & $4(22.2 \%)$ & $12(14.1 \%)$ & $1.66(0.14-18.87)$ & 0.68 \\
\hline Head and Neck & $4(3.8 \%)$ & $1(5.6 \%)$ & $3(3.5 \%)$ & $1.66(0.07-37.72)$ & 0.74 \\
\hline Others & $5(4.9 \%)$ & $1(5.6 \%)$ & $4(4.7 \%)$ & Reference & \\
\hline Stage & & & & $4.04(1.09-15.1)$ & 0.04 \\
\hline Metastatic & $62(60.2 \%)$ & 15 (83.3\%) & 47 (55.3\%) & & \\
\hline Non-metastatic & $41(39.8 \%)$ & $3(16.7 \%)$ & 38 (44.7\%) & & \\
\hline Treatment options & & & & & 0.10 \\
\hline No treatment & $15(14.6 \%)$ & $5(27.8 \%)$ & $10(11.8 \%)$ & Reference & \\
\hline Adjuvant/neoadjuvant & $36(35 \%)$ & $3(16.6 \%)$ & $33(38.8 \%)$ & $0.17(0.03-0.87)$ & 0.03 \\
\hline Palliative & $52(50.4 \%)$ & $10(55.6 \%)$ & $42(49.4 \%)$ & $0.47(0.13-1.70)$ & 0.25 \\
\hline Anti-Tumoral agents (n: 88) & & & & & 0.77 \\
\hline Chemotherapy & $54(62.1 \%)$ & $7(58.3 \%)$ & 47 (62.7\%) & Reference & \\
\hline TKi & $10(11.5 \%)$ & $1(8.3 \%)$ & $9(12 \%)$ & $0.67(0.07-6.08)$ & 0.72 \\
\hline Immunotherapy & $3(3.4 \%)$ & $1(8.3 \%)$ & $2(2.7 \%)$ & $3.35(0.26-42.07)$ & 0.34 \\
\hline Hormonal therapy & $20(23 \%)$ & $3(25 \%)$ & $17(22.6 \%)$ & $1.18(0.27-5.11)$ & 0.82 \\
\hline
\end{tabular}

* independent samples T-test.

higher in the non-survivors than in the survivors. In an evaluation of the association between mortality risk and the presence of metastatic disease, the mortality rate was found to be higher in patients with metastatic disease [24.2\% in metastatic patients, $7.3 \%$ in non-metastatic patients (HR: $4.04,95 \% \mathrm{Cl}(1.09-15.1)$, $(p=0.04)$ ]. Of the patients, $52 \%$ ( $n: 52)$ were undergoing palliative chemotherapy and $36 \%$ ( $n$ : 37 ) adjuvant/neoadjuvant therapy, while $14.6 \%$ ( $n: 15)$ were receiving no treatment. When the sample was evaluated based on treatment modalities, survival was better for COVID-19 positive patients receiving adjuvant/neoadjuvant therapy than for the group receiving no treatment [HR: $0.17,95 \% \mathrm{Cl}(0.03-0.87),(p=0.03)]$. There was no statistically significant difference in the mortality rates of those receiving palliative chemotherapy and those undergoing no treatment [HR: $0.47,95 \% \mathrm{Cl}(0.13-1.70)$, $(p=0.25)]$. Of the 87 patients receiving treatment, $62.1 \%$ (n: 54 ) were receiving chemotherapy, 23\% (n: 20) hormonal therapies, $11.5 \%$ ( $n: 10)$ tyrosine kinase inhibitors and $3.4 \%(n: 3)$ immunotherapy agents. When the treatments of the patients were compared, no statistically significant difference was found in the mortality rates associated with the different therapeutic agents $(p=0.77)$.

When the total 1,454 patients were evaluated in terms of hepatitis B immunization, data was available for 92 patients with a COVID-19 diagnosis and 1,362 pa- 


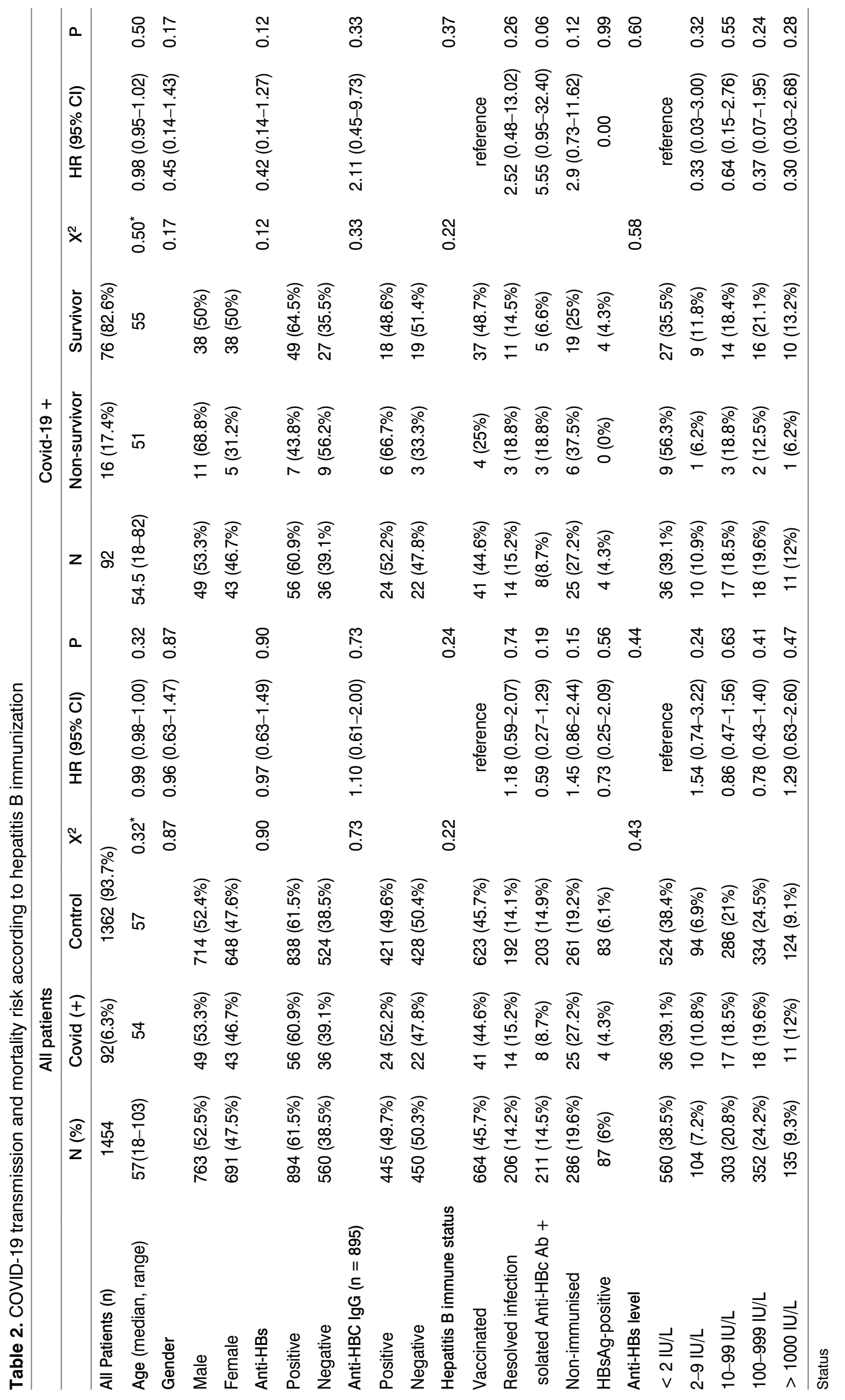


Table 3. Multivariate analysis for mortality risk in patients with COVID-19

\begin{tabular}{lcc}
\hline & \multicolumn{2}{c}{ Multivariate analysis } \\
\hline Age & HR (95\% Cl) & P-value \\
Gender (male/female) & $0.96(0.91-1.01)$ & 0.20 \\
Comorbidities (no/yes) & $0.43(0.11-1.71)$ & 0.23 \\
Stage (metastatic/ non-metastatic) & $2.00(0.41-9.73)$ & 0.39 \\
Treatment options & $11.16(1.19-104.6)$ & 0.03 \\
No treatment & & 0.06 \\
Adjuvant/neoadjuvant & reference & 0.45 \\
Palliative & $0.43(0.05-3.79)$ & 0.02 \\
Hepatitis B immunization (no/yes) & $0.10(0.01-0.69)$ & 0.21 \\
\hline
\end{tabular}

tients in the control group. The detailed clinical and laboratory findings of the patients related to hepatitis $B$ are presented in Table 2 . The seroprevalence of hepatitis B $(\mathrm{HbsAg}+)$ was $6 \%(87 / 1454)$ among the study patients. In the case group, $4.3 \%$ (n: 4) of patients had HbsAg positivity, $60.9 \%$ (n: 56 ) had anti-Hbs positivity and $52.2 \%$ (n: 24 ) had anti-HBc IgG positivity. In the control group, $6.1 \%$ ( $\mathrm{n}: 83$ ) of the patients had $\mathrm{HbsAg}$ positivity, $61.5 \%$ (n: 838 ) had anti-Hbs positivity and 49.6\% (n: 421) had anti-Hbc IgG positivity. The mean anti-Hbs level was $10 \mathrm{lU} / \mathrm{L}$ in the case group compared with $18.2 \mathrm{IU} / \mathrm{L}$ in the control group ( $p=0.66$ ). When the immunization status of the patients was analysed, $44.6 \%$ ( $n: 41$ ) of the patients were found to be vaccinated, $15.2 \%$ ( $n: 14)$ had resolved infection, $8.7 \%$ (n: 8) had isolated anti-Hbc IgG antibody positivity, $27.2 \%$ (n: 25) were non-immunized and 4.3\% (n: 4) were HbsAg positive in the case group. In the control group, in turn, $45.7 \%$ ( $n: 623$ ) were vaccinated, $14.1 \%$ ( $n: 192)$ had resolved infection, $14.9 \%$ ( $\mathrm{n}: 203)$ had isolated anti-Hbc IgG antibody positivity, 19.2\% (n: 261) were non-immunized, and $6.1 \%$ (n: 83 ) were HbsAg positive. When the case and control groups were compared according to these five categories, there was no statistically significant difference in the risk of COVID-19 transmission $(p=0.22)$ or the risk of mortality $(p=0.22)$ between the groups. When the patients' anti-Hbs antibody levels (< 2 IU/L, 2-9 IU/L, 10-99 IU/L, 100-999 IU/L and $>1000 \mathrm{IU} / \mathrm{L})$ were categorized, no statistically significant difference was found between the two groups in the risk of COVID-19 transmission ( $p=0.43$ ) and the risk of mortality ( $p=0.53$ ) based on antibody levels.

Age, gender, presence of comorbidities, stage of the disease, treatment options, hepatitis $B$ immunization status were examined with univariate and multivariate analyses. The results of the analyses are presented in Table 3. The multivariate analysis identified palliative chemotherapy [HR: $0.1,95 \% \mathrm{Cl}(0.01-0.69),(p=0.02)$ ] and stage [HR: $0.09,95 \% \mathrm{Cl}(0.01-0.83),(p=0.03)]$ as independent prognostic factors for mortality risk in patients with COVID-19 infection as univariate analysis did not indicate palliative treatment as a prognostic factor.

\section{Discussion}

Among the different patient groups, cancer patients are at one of the greatest risks from the COVID-19 pandemic [1-3]. The present study investigates the effect of the characteristic and clinical outcomes of cancer patients who presented to the authors' clinic during the COVID-19 pandemic related to COVID-19 transmission risk and mortality, intending to identify the associated risk factors.

Among the 1,515 patients who presented to the clinic during the pandemic, $10.1 \%$ (n: 153) were diagnosed with COVID-19, which is a rate considerably higher than reported in COVID-19 incidence studies conducted with cancer patients $(10.1 \%$ vs. $0.79-1.3 \%)$ [10, 11$]$. This may be attributed to the fact that the reported studies were carried out during the early stages of the pandemic, and that cases increased afterwards, and that a COVID-19 test was administered routinely to the patients before treatment following the recommended guidelines later in the pandemic, leading to the detection also of asymptomatic cases.

The Southeastern Anatolia Region of Turkey, where the oncology centre is located, is an endemic area for hepatitis B infection (7\%, with a high prevalence of HBsAg) [12], and so all patients referring to the oncology centre are routinely checked for hepatitis B serology with Elisa before treatment planning. Chemotherapy-induced immunosuppression may cause $\mathrm{HBV}$ reactivation in active or inactive HBV carriers, and HBV reactivation during anti-tumour therapy can lead to life-threatening clinical manifestations (fulminant 
hepatitis, hepatic failure, and mortality) in addition to discontinuation of anti-tumour therapies [13]. Guidelines recommend HBV screening for treatments with severe immunosuppressive activity or patients at high risk of HBV infection, and prophylactic antiviral therapy in cancer patients at high risk of HBV reactivation [12, 13]. In the present study, $34.7 \%$ of cases had encountered hepatitis $B$ at some point in their lives, and $6 \%$ had $\mathrm{HbsAg}$ positivity. The seroprevalence of hepatitis $\mathrm{B}$ among patients was consistent with the prevalence of hepatitis $B$ in the study region ( $6 \%$ vs. $7 \%$ ), and there was no significant difference in $\mathrm{HbsAg}$ positivity between the patients with COVID-19 and the control group [4.3\% vs. $6.1 \%(p=0.56)]$.

Whether or not hepatitis B seropositivity and immunization status affect the course and outcomes of COVID-19 infection are yet to be confirmed. Chronic HBV infection may affect the likelihood of developing clinically important infectious manifestation with COVID-19 [13-15]. A review article suggested that chronic HBV infection may contribute to a decrease in virus-specific T-cell activity, and indirectly to the intensity of the cytokine storm [16]. It is also hypothesized that immunization with hepatitis $A$, hepatitis $B$ and $B C G$ vaccines can provide a protective effect against COVID-19 through immune maturation [6]. That said, in an animal study investigating whether childhood vaccines, including hepatitis $B$ and BCG, produced cross-reactive neutralizing antibodies against SARSCoV-2, none were found to produce antibodies against SARS-CoV-2 after vaccination [17]. In the present study, when the immunization profiles (vaccinated, resolved infection, isolated anti-HBc $\mathrm{Ab}(+)$, non-immunized and HBsAg-positive) developed against hepatitis $B$ were compared between the case and control groups, no significant difference was noted in the risk of COVID-19 transmission between the groups $(p=0.22)$, and no significant difference was noted either in mortality risk between the different immunization profiles of COVID-19-positive patients $(p=0.37)$. It was further observed that anti-Hbs serum levels had no effect on COVID-19 transmission $(p=0.44)$ or mortality $(p=0.60)$.

COVID-19 infections can lead to impaired liver function and severe liver injury [18]. A meta-analysis of studies evaluating the hepatic findings of COVID-19 reported an HBV prevalence of 0.9\% [19]. The prevalence of HBV infection was found to be $6.1 \%$ and $4.3 \%$ in the study control group patients with a cancer diagnosis and cancer patients with COVID-19, respectively. Although these rates seem higher than those reported in the literature, they were close to the prevalence $(7 \%)$ of the endemic region in the general population. In a previous study evaluating the clinical characteristics of patients with hepatitis B and COVID-19 coinfection, 20 (6.1\%) of 326 COVID-19 positive patients had concurrent HBV infection. The study found no significant difference in discharge rates, length of hospital stays, exacerbation of liver injury, and mortality between the patients with and without hepatitis B infection [20].

Among cancer patients, those with thoracic malignancies such as lung cancer are considered at high risk of COVID-19 mortality due to associated age, comorbidities, history of smoking and existing pulmonary damage, in addition to the treatments administered for the disease [21]. In the present study, lung cancer was the fourth most common form of cancer after breast, gastrointestinal system (GIS) and genitourinary system (GUS) cancers, among the study patients with COVID-19 infection. However, lung cancers did not differ from the other cancer types in terms of COVID-19-related mortality risk $(p=0.68)$.

In the present study, multivariate analysis showed that the presence of metastatic disease and the receipt of palliative chemotherapy were statistically different between the survivors and non-survivors among the cancer patients with COVID-19 infection as univariate analysis did not indicate palliative treatment as a prognostic factor. Survival among the COVID-19 positive patients receiving palliative therapy was better than in those receiving no treatment [HR: $0.1,95 \% \mathrm{Cl}(0.01-$ $0.69),(p=0.02)$ ]. Most patients in the group receiving no treatment were in remission. Although this would appear to be contradictory, it actually recalls the idea that chemotherapy can suppress possible excessive immune reactions (cytokine storm) due to COVID-19 and can provide a survival benefit to the patients. In this regard, a previous study comparing the immune response to SARS-CoV-2 in cancer patients undergoing active antitumor therapy with the immune response in healthy individuals reported differences in immune cell profiles, indicating different inflammatory responses in the two groups. The study suggested that cancer treatments such as chemotherapy, biological agents and immunotherapies may affect the body's immune response against COVID-19, leading to a milder manifestation of the infection in cancer patients with COVID-19 infection [22]. Also, fewer myelosuppressive therapeutic agents were preferred for palliative chemotherapies, and dose reductions and delays were applied more frequently during the pandemic, following the recommended guidelines [23]. In contrast, granulocyte colony-stimulating factor (GCSF) support was more often preferred for the minimization of the risk of myelosuppression. Likely, these adaptive practices may also influence the study findings.

The present study was unable to identify any statistical differences in the treatments of the survivors and non-survivors diagnosed with COVID-19. The potential 
interaction between oncological therapeutic agents and COVID-19 infection is not fully known [24], although the TERAVOLT study, which investigated the risk factors associated with mortality in patients with thoracic malignancies and COVID-19 infections and presented at ASCO 2020's meeting (American society of clinical oncology 2020), reported the treatment of cancer patients with chemotherapy to be a risk factor for mortality associated with COVID-19 infection, while treatment with tyrosine kinase inhibitors and immunotherapy was not a risk factor. The same study further reported advanced age ( $>65$ years), comorbidities, performance status $>1$, and receiving steroid or anticoagulant therapy to be risk factors associated with mortality [21]. In a comparison of the patients in the present study in terms of the applied anti-tumour therapies (chemotherapy, immunotherapy, tyrosine kinase inhibitor and hormonal therapies), it was observed that types of anti-tumour therapy did not affect mortality in patients with COVID-19 infection. In a study conducted in the early stages of the pandemic that evaluated cancer patients with COVID-19 infection, the receipt of chemotherapy within four weeks of the emergence of COVID-19 infection symptoms and male gender were reported to be poor prognostic factors. In addition, hepatitis $B$ virus infection was detected in $6 \%$ of the 205 patients in the study, although the same study observed that the presence of hepatitis $B$ virus infection was not among the risk factors for mortality [25]. A study by Lee et al., in turn, reported the receipt of chemotherapy within the last four weeks to have no significant effect on COVID-19 mortality, while advanced age and the presence of cardiovascular comorbidities were high-risk factors [26]. Cancer patients undergoing active treatment have been referred to as high-risk patients since the beginning of the pandemic. After viewing the findings of different studies, it is expected that the potential roles of cancer and cancer treatments during COVID-19 will be clarified through further studies.

The limitations of this study include its single-centre and retrospective design, the heterogeneous patient population in terms of diagnosis, stage and treatments, and the lack of information about COVID-19 treatments (such as steroids, etc.) that might have an impact on mortality risk. Moreover, the fact that the study was carried out in one particular region in the country, and that the patient group had other diseases, may have influenced the study findings. Finally, some of the control group patients may contract COVID-19 in the future, which is another study limitation.

\section{Conclusions}

In conclusion, this study is the first to evaluate the relationship between COVID-19 and hepatitis B immunization in cancer patients. The present study identified the presence of metastatic disease and the receipt of palliative chemotherapy as negative and positive prognostic factors, respectively, but no statistically significant relationship between the risk of COVID-19 transmission and mortality, and hepatitis $B$ immunization status. Factors other than hepatitis $B$ immunization status are likely to be more effective in the risk of COVID-19 transmission and mortality among cancer patients.

\section{Funding: This study was not supported by any} organization or entity. The authors have no financial involvement with any organization or entity. No writing assistance was utilized in the production of this manuscript.

Ethical permissions: The study was conducted in compliance with the ethical principles according to the Declaration of Helsinki. Special approval was obtained from the Ministry of Health for this study.

\section{References}

1. Kuderer NM, Choueiri TK, Shah DP, et al. COVID-19 and Cancer Consortium. Clinical impact of COVID-19 on patients with cancer (CCC19): a cohort study. Lancet. 2020; 395(10241): 1907-1918, doi: 10.1016/S0140-6736(20)31187-9, indexed in Pubmed: 32473681.

2. Dai M, Liu D, Liu M, et al. Patients with Cancer Appear More Vulnerable to SARS-CoV-2: A Multicenter Study during the COVID-19 Outbreak. Cancer Discov. 2020; 10(6): 783-791, doi: 10.1158/2159-8290.CD20-0422, indexed in Pubmed: 32345594.

3. Ofori-Asenso R, Ogundipe O, Agyeman AA, et al. Cancer is associated with severe disease in COVID-19 patients: a systematic review and meta-analysis. Ecancermedicalscience. 2020; 14: 1047, doi: 10.3332/ecancer.2020.1047, indexed in Pubmed: 32565900.

4. Lewis MA. Between Scylla and Charybdis - Oncologic Decision Making in the Time of Covid-19. N Engl J Med. 2020; 382(24): 2285-2287, doi: 10.1056/NEJMp2006588, indexed in Pubmed: 32267650.

5. Liu Y, Mao B, Liang S, et al. Shanghai Clinical Treatment Experts Group for COVID-19. Association between age and clinical characteristics and outcomes of COVID-19. Eur Respir J. 2020; 55(5), doi: 10.1183/13993003.01112-2020, indexed in Pubmed: 32312864.

6. Lyu J, Miao T, Dong J, et al. Reflection on lower rates of COVID-19 in children: Does childhood immunizations offer unexpected protection? Med Hypotheses. 2020; 143: 109842, doi: 10.1016/j. mehy.2020.109842, indexed in Pubmed: 32425304.

7. Salman S, Salem ML. Routine childhood immunization may protect against COVID-19. Med Hypotheses. 2020 [Epub ahead of print]; 140: 109689, doi: 10.1016/j.mehy.2020.109689, indexed in Pubmed: 32240961.

8. Klinger D, Blass I, Rappoport N, et al. Significantly Improved COVID-19 Outcomes in Countries with Higher BCG Vaccination Coverage: A Multivariable Analysis. Vaccines (Basel). 2020; 8(3), doi: 10.3390/vaccines8030378, indexed in Pubmed: 32664505.

9. Escobar LE, Molina-Cruz A, Barillas-Mury C. BCG vaccine protection from severe coronavirus disease 2019 (COVID-19). Proc Natl Acad Sci U S A. 2020; 117(30): 17720-17726, doi: 10.1073/pnas.2008410117, indexed in Pubmed: 32647056

10. Yu J, Ouyang W, Chua MLK, et al. SARS-CoV-2 Transmission in Patients With Cancer at a Tertiary Care Hospital in Wuhan, China. JAMA Oncol. 2020; 6(7): 1108-1110, doi: 10.1001/jamaoncol.2020.0980, indexed in Pubmed: 32211820.

11. Bertuzzi AF, Marrari A, Gennaro N, et al. Low Incidence of SARS-CoV-2 in Patients with Solid Tumours on Active Treatment: An Observational Study at a Tertiary Cancer Centre in Lombardy, Italy. Cancers (Basel). 2020; 12(9), doi: 10.3390/cancers12092352, indexed in Pubmed: 32825295. 
12. Tozun N, Ozdogan O, Cakaloglu Y, et al. Seroprevalence of hepatitis B and $\mathrm{C}$ virus infections and risk factors in Turkey: a fieldwork TURHEP study. Clin Microbiol Infect. 2015; 21(11): 1020-1026, doi: 10.1016/j. cmi.2015.06.028, indexed in Pubmed: 26163105.

13. Bozza $C$, Cinausero $M$, lacono D, et al. Hepatitis B and cancer: A practical guide for the oncologist. Crit Rev Oncol Hematol. 2016; 98 : 137-146, doi: 10.1016/j.critrevonc.2015.10.017, indexed in Pubmed: 26657667.

14. Cheung KS, Seto WK, Lai CL, et al. Prevention and management of hepatitis B virus reactivation in cancer patients. Hepatol Int. 2016; 10(3): 407-414, doi: 10.1007/s12072-015-9692-3, indexed in Pubmed: 26739135

15. European Association For The Study Of The Liver. EASL clinical practice guidelines: Management of chronic hepatitis $B$ virus infection. J Hepatol. 2012; 57(1): 167-185, doi: 10.1016/j.jhep.2012.02.010, indexed in Pubmed: 22436845.

16. Anugwom CM, Aby ES, Debes JD. Inverse Association Between Chronic Hepatitis B Infection and Coronavirus Disease 2019 (COVID-19): Immune Exhaustion or Coincidence? Clin Infect Dis. 2021; 72(1): 180-182, doi: 10.1093/cid/ciaa592, indexed in Pubmed: 32502247.

17. Kandeil A, Gomaa MR, El Taweel A, et al. Common childhood vaccines do not elicit a cross-reactive antibody response against SARSCoV-2. PLoS One. 2020; 15(10): e0241471, doi: 10.1371/journal. pone.0241471, indexed in Pubmed: 33112930

18. Zhang C, Shi L, Wang FS. Liver injury in COVID-19: management and challenges. Lancet Gastroenterol Hepatol. 2020; 5(5): 428-430, doi: 10.1016/S2468-1253(20)30057-1, indexed in Pubmed: 32145190.

19. Kunutsor SK, Laukkanen JA. Hepatic manifestations and complications of COVID-19: A systematic review and meta-analysis. J Infect. 2020; 81(3) e72-e74, doi: 10.1016/j.jinf.2020.06.043, indexed in Pubmed: 32579984.
20. Chen L, Huang S, Yang J, et al. Clinical characteristics in patients with SARS-CoV-2/HBV co-infection. J Viral Hepat. 2020; 27(12): 1504-1507, doi: 10.1111/jvh.13362, indexed in Pubmed: 32668494

21. Garassino MC, Whisenant JG, Huang LC, et al. TERAVOLT investigators. COVID-19 in patients with thoracic malignancies (TERAVOLT) first results of an international, registry-based, cohort study. Lancet Oncol. 2020; 21(7): 914-922, doi: 10.1016/S1470-2045(20)30314-4, indexed in Pubmed: 32539942

22. Goshen-Lago T, Szwarcwort-Cohen M, Benguigui M, et al. The Potential Role of Immune Alteration in the Cancer-COVID19 Equation-A Prospective Longitudinal Study. Cancers (Basel). 2020; 12(9), doi: 10.3390/cancers12092421, indexed in Pubmed: 32859016

23. European Society of Medical Oncology (ESMO). Cancer Patient Management During The COVID-19 Pandemic. https://www. esmo. org/guidelines/cancer-patient-management-during-the-covid-19pandemic (April 10,2020).

24. Bersanelli M. Controversies about COVID-19 and anticancer treatment with immune checkpoint inhibitors. Immunotherapy. 2020; 12(5): 269-273, doi: 10.2217/imt-2020-0067, indexed in Pubmed: 32212881

25. Yang $\mathrm{K}$, Sheng $\mathrm{Y}$, Huang $\mathrm{C}$, et al. Clinical characteristics, outcomes, and risk factors for mortality in patients with cancer and COVID-19 in Hubei, China: a multicentre, retrospective, cohort study. Lancet Oncol. 2020; 21 (7): 904-913, doi: 10.1016/S1470-2045(20)30310-7, indexed in Pubmed: 32479787

26. Lee LYW, Cazier JB, Starkey T, et al. UK Coronavirus Cancer Monitoring Project Team, UK Coronavirus Monitoring Project Team. COVID-19 mortality in patients with cancer on chemotherapy or other anticancer treatments: a prospective cohort study. Lancet. 2020; 395(10241): 1919-1926, doi: 10.1016/S0140-6736(20)31173-9, indexed in Pubmed: 32473682 